\title{
Coupling finite elements and particles for adaptivity: an application to consistently stabilized convection-diffusion
}

\author{
Sonia Fernández-Méndez and Antonio Huerta \\ Departament de Matemàtica Aplicada III, E.T.S. de Ingenieros de Caminos, \\ Universitat Politècnica de Catalunya, Jordi Girona 1, E-08034 Barcelona, Spain
}

\begin{abstract}
A mixed approximation coupling finite elements and mesh-less methods is presented. It allows selective refinement of the finite element solution without remeshing cost. The distribution of particles can be arbitrary. Continuity and consistency is preserved. The behaviour of the mixed interpolation in the resolution of the convection-diffusion equation is analyzed.
\end{abstract}

\section{Introduction}

Mesh-less or particle methods such as reproducing kernel particle methods (RKPM) [12], element-free Galerkin (EFG) [1,4] or smooth particle hydrodynamics (SPH) [5], among others (see [3,11] for a general presentation), have nowadays proven their applicability in computational mechanics. They do not require to generate a mesh (a connectivity matrix) and thus, they are specially suited for certain problems, for instance adaptive refinement computations. However, particle methods based on a weak formulation, such as EFG or RKPM, suffer from an important computational cost. This is mainly due to the need of a very accurate integration quadrature for the computation of the weak form.

On the other hand, from a practical point of view, finite elements are less costly and implement Dirichlet boundary conditions in a simple way. However, the relative cost of the mesh generation process is, for some problems, very large. In particular, the cost of remeshing in adaptive refinement problems is clearly not negligible.

Several authors have already proposed to mix finite elements and meshfree interpolations. The objective is always to use the advantages of each method. In [4] finite elements are used near the Dirichlet boundaries and EFG is employed in the interior of the computational domain. This simplifies considerably the prescription of essential boundary conditions. A mixed interpolation is proposed in the transition region: area where both finite elements and particles have an influence. This mixed interpolation requires the substitution of finite element nodes by particles and the definition of ramp functions. Thus the transition is of the size of one finite element, the interpolation in linear and the position of the particles is not arbitrary. With the 
same objectives in [7] the finite element domain and the meshless region are coupled using Lagrange multipliers.

Here a new formulation is presented. It can handle any order of interpolation, does not need the ramp functions, and does not require the substitution of nodes by particles. That is, as many particles can be added where they are needed independently of the adjacent finite element mesh. In fact, the proposed interpolation can be seen as a particular case of a more general formulation that allows both enrichment and coupling of finite elements with particles, see [8] for details. This paper is focused on coupling. This is done preserving continuity of the solution and enforcing uniform consistency for the mixed interpolation. The formulation is developed for the EFG method. However, its generalization to other particle methods such as RKPM is straight forward.

This mixed interpolation combining finite elements and EFG turns out useful in adaptive refinement processes. In fact, the adaptive process would be: (1) compute an approximation with a finite element mesh, (2) do an a posteriori error estimation and (3) improve the solution with no remeshing cost taking off some nodes and adding a suitable distribution of particles. In the following sections the formulation is developed, the applicability conditions


are discus

Finall equation lows to ca dard Gale boundary


finite element methods are usually non consistently stabilized. The second (or higher) order derivatives needed in the stabilization term are usually ne-



parent and some of them have been explored [10]. This ensures the theoretical convergence rates, but the prize is to drastically increase the computational cost and the implementation difficulty. In EFG second derivatives are defined continuously in the whole domain and are computed at a reduced extra cost [2], that is an extra cost negligeable compared with finite elements. There are no neglected terms in the weak form and the consistency of the formulation is preserved. Therefore, the use of EFG for the discretization refinement in boundary or internal layers will also allow the use of a consistently stabilized formulation in these regions.

\section{Fundamentals of Element-Free Galerkin}

Mesh-less methods are based in a functional interpolation of the form:

$$
u(x) \simeq u^{\rho}(x)=\sum_{j \in I^{\rho}} u\left(x_{j}\right) N_{j}^{\rho}(x)
$$


given a number of particles $\left\{x_{j}\right\}_{j \in I^{\rho}}$ in the domain $\Omega \subset \mathbb{R}^{n}$. In the context of the EFG method [1,4], the interpolation (shape) functions, $N_{j}^{\rho}(x)$, are defined as:

$$
N_{j}^{\rho}(x)=\mathbf{P}^{T}\left(x_{j}\right) \alpha(x) \phi\left(\frac{x-x_{j}}{\rho}\right),
$$

where $\mathbf{P}^{T}(x)=\left\{p_{0}(x), p_{2}(x), \ldots, p_{l}(x)\right\}$ includes a complete basis of the subspace of polynomials of degree $m$ and the vector $\alpha(x)$ in $\mathbb{R}^{l+1}$ is unknown. In one dimension, it is usual that $p_{i}(x)$ coincides with the monomials $x^{i}$, and, in this particular case, $l=m$. The function $\phi(x)$ is a weighting function (positive, even and with compact support) which characterizes the mesh-less method. For instance, if $\phi(x)$ is continuous together with its first $k$ derivatives, the interpolation is also continuous together with its first $k$ derivatives [12]. In (2) the weighting function has been translated, centered in $x_{j}$, and its support scaled by the dilation parameter $\rho$. The unknown vector $\alpha(x)$ is determined imposing the so-called reproducibility or consistency condition. This condition imposes that $u^{\rho}(x)$ defined in (1) interpolates exactly polynomials of degree less or equal $m$, i.e.


(3)
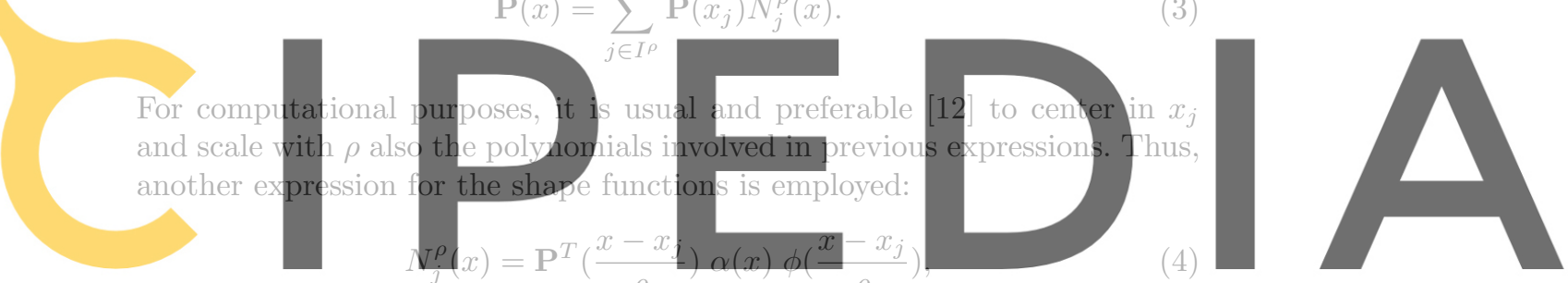



$$
\mathrm{P}(0)=\sum_{j \in I^{\rho}} \mathrm{P}\left(\frac{x-x_{j}}{\rho}\right) N_{j}^{\rho}(x),
$$

which is equivalent to condition (3) when $\rho$ is constant everywhere. After substitution of (4) in (5) the usual linear system of equations, that determines $\alpha(x)$, is obtained:

$$
\mathbf{M}(x) \alpha(x)=\mathbf{P}(0)
$$

with

$$
\mathbf{M}(x)=\sum_{j \in I^{\rho}} \mathbf{P}\left(\frac{x-x_{j}}{\rho}\right) \mathbf{P}^{T}\left(\frac{x-x_{j}}{\rho}\right) \phi\left(\frac{x-x_{j}}{\rho}\right) .
$$

Notice that for each $x$ in $\mathbb{R}^{n}$ the previous sum only involves those particles whose support - the support of the weighting function $\phi$ - includes $x$.

Section 3.2 presents a discussion on the necessary conditions for $\mathbf{M}(x)$ to be positive definite, namely, the requirements on the particle distribution and the value of the dilation parameter. This will allow to compute the vector $\alpha$ at each point and thus determine the shape functions, $N_{j}^{\rho}(x)$. 
Remark 1. The consistency conditions (3) and (5) are equivalent if the dilation parameter $\rho$ is constant. When the dilation parameter varies at each particle another definition of the shape functions is recommended

$$
N_{j}^{\rho}(x)=\mathbf{P}^{T}\left(\frac{x-x_{j}}{\rho}\right) \alpha(x) \phi\left(\frac{x-x_{j}}{\rho_{j}}\right),
$$

where $\rho_{j}$ is the dilation parameter associated to particle $x_{j}$, and a constant $\rho$ is employed in the scaling of the polynomials $\mathbf{P}$.

Remark 2. The dilation parameter $\rho$ characterizes the support of the shape functions $N_{i}^{\rho}(x)$. In fact, it plays a role similar to the element size in the finite element method. An $h$-refinement in finite elements can be produced in meshless methods decreasing proportionally the dilation parameter $\rho$ and the distance between particles. Convergence of EFG is discussed in [12] as a particular case of RKPM. The a priori bound is very similar to the bound in finite elements. The parameter $\rho$ plays the role of $h$, and $m$ (the order of consistency) plays the role of the degree of the interpolation polynomials in the finite element mesh.


$$
u^{h}(x)=\sum u\left(x_{i}\right) N_{i}^{h}(x)
$$

Register for free at https//www.scipedia.comito download the version without the watermark

There is also a set of particles $\left\{x_{j}\right\}_{j \in I^{\rho}}$ with their associated interpolation functions $N_{j}^{\rho}(x)$, that are going to take care of the meshless contribution,

$$
u^{\rho}(x)=\sum_{j \in I^{\rho}} u\left(x_{j}\right) N_{j}^{\rho}(x) .
$$

The computational domain $\Omega$ is divided in three non disjoint regions: one where finite elements have an influence, $\Omega^{h}$, another where particles have an influence, $\Omega^{\rho}$, and finally, one transition region, $\widetilde{\Omega}=\overline{\Omega^{h} \cap \Omega^{\rho}}$, where both finite elements and particles take care of the interpolation, see figure 1 . As will be seen in the examples, such a situation may be of interest if a computation with finite elements needs to be refined in a region $\Omega^{\rho}$ without remeshing. The nodes of the original finite element mesh are removed in $\Omega^{\rho}$ but as many particles as needed are added in that region.

In the region where only finite elements are present, $\Omega^{h} \backslash \Omega^{\rho}$, a standard, and thus consistent, finite element approximation is considered,

$$
u(x) \simeq u^{h}(x) \quad \text { in } \Omega^{h} \backslash \Omega^{\rho} .
$$




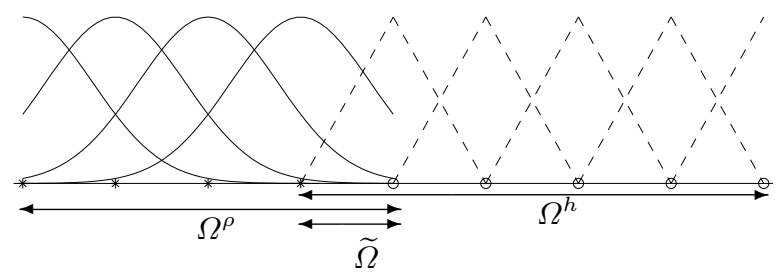

Fig. 1. FE and EFG shape functions before imposing consistency

In the region where only particles have an influence, $\Omega^{\rho} \backslash \Omega^{h}$, the standard, and thus consistent, EFG approximation is considered,

$$
u(x) \simeq u^{\rho}(x) \quad \text { in } \Omega^{\rho} \backslash \Omega^{h} .
$$

However, in the area where both interpolations have an influence, $\widetilde{\Omega}$, a mixed interpolation must be defined

$$
u(x) \simeq u^{h}(x)+u^{\rho}(x) \quad \text { in } \widetilde{\Omega} .
$$



The objective now is to develop a mixed functional interpolation, such


3.1 Evaluation of the mesh-free shape functions $N_{j}^{\rho}$


of (8) and (9) into (12), namely

$$
u(x) \simeq \sum_{i \in I^{h}} u\left(x_{i}\right) N_{i}^{h}(x)+\sum_{j \in I^{\rho}} u\left(x_{j}\right) N_{j}^{\rho}(x) .
$$

Where $N^{\rho}(x)$ is defined, as previously, in (4). And, as before, the vector of unknown functions, $\alpha(x)$, is determined using the consistency condition. Now the reproducibility conditions impose that (13) must interpolate exactly a complete basis of polynomials of order less or equal to $m$. That is,

$$
\mathbf{P}(0)=\sum_{j \in I^{\rho}} \mathbf{P}\left(\frac{x-x_{j}}{\rho}\right) N_{j}^{\rho}(x)+\sum_{i \in I^{h}} \mathbf{P}\left(\frac{x-x_{i}}{\rho}\right) N_{i}^{h}(x),
$$

which is the natural extension of (5). The linear system of equations that determines $\alpha$ is obtained by substitution of (4) in (14):

$$
\mathbf{M}(x) \alpha(x)=\mathbf{P}(0)-\sum_{i \in I^{h}} \mathbf{P}\left(\frac{x-x_{i}}{\rho}\right) N_{i}^{h}(x) .
$$






Fig. 2. Approximation functions before and after imposing the consistency condition of order one.

The least-squares matrix $\mathbf{M}$ is identical to the matrix employed in the standard EFG method, Eq. (7).

Proposition 1. The approximation $u^{h}+u^{\rho}$ is continuous in $\Omega$ if:

1. the same order of consistency $m$ is imposed all over $\Omega$ (i.e. $m$ coincides with the degree of the FE base), and

2. the domain of influence of particles, $\Omega^{\rho}$, coincides exactly with the region where finite elements do not have a complete basis.

Proof. The approximation $u^{h}+u^{\rho}$ is continuous as long as the shape functions $N_{j}^{\rho}$ are continuous. Moreover, by definition, the approximation is continuous in $\Omega^{h} \backslash \Omega^{\rho}$, in $\Omega^{\rho} \backslash \Omega^{h}$ and in the interior of the transition region $\widetilde{\Omega}$, see equa-



tions $(10)$

in $\partial \widetilde{\Omega}$. Or

First,

are chopp

$\partial \widetilde{\Omega} \cap \Omega^{h}$

and of order $m$. Tn


equal to $m$ are interpolated exactly. Thus, it is easy to verify that



Recalling (15), the previous equation implies that $\alpha(x)=\mathbf{0}$, and consequently, $N_{j}^{\rho}(x)=0$ in $\partial \widetilde{\Omega} \cap \Omega^{h}$, see equation (4).

On the other hand, recall that along $\partial \widetilde{\Omega} \cap \Omega^{\rho}$ as well as in $\Omega^{\rho} \backslash \Omega^{h}$, by definition, finite elements have no influence, i.e.

$$
\mathbf{P}(0)-\sum_{i \in I^{h}} \mathbf{P}\left(\frac{x-x_{i}}{\rho}\right) N_{i}^{h}(x)=\mathbf{P}(0) .
$$

Therefore, in $\partial \widetilde{\Omega} \cap \Omega^{\rho}$ the $N_{j}^{\rho}$ are identical to the standard EFG ones, and the continuity in $\partial \widetilde{\Omega} \cap \Omega^{\rho}$ is proved.

Note that condition 2 in this proposition means that no particles are added in "complete" finite elements, i.e. elements where no node has been suppressed. Moreover, weighting functions $\phi$ are chopped off in those "complete" finite elements, see figure 2 . In other words, $\Omega^{\rho}$ is the union of elements where at least one node has been removed. 


\subsection{Admissible particle distribution}

As in standard EFG, matrix $\mathbf{M}(x)$ must be regular (invertible) everywhere, i.e. at each point $x \in \widetilde{\Omega}$. Only the right hand side of (15) differs from the EFG system of equations, Eq. (6). Thus, as in EFG, the number of particles, their position and their related dilation parameters can not be taken arbitrarily [12]. At a point $x \in \Omega^{\rho}$, matrix $\mathbf{M}(x)$ can be viewed as a Gram matrix defined with the discrete scalar product

$$
<f, g>_{x}=\sum_{j \in I_{x}^{\rho}} f\left(\frac{x-x_{j}}{\rho}\right) g\left(\frac{x-x_{j}}{\rho}\right) \phi\left(\frac{x-x_{j}}{\rho}\right),
$$

where $I_{x}^{\rho}:=\left\{j \in I^{\rho}\right.$ such that $\left.\left|x_{j}-x\right| \leq \rho\right\}$, and with the linear independent polynomials in $\mathbf{P}(z)$. If the scalar product, $\langle\cdot, \cdot\rangle_{x}$, is degenerated the matrix $\mathbf{M}(x)$ is singular. The regularity of $\mathbf{M}(x)$ is ensured if enough particles are in the neighborhood of every point $x$ and they are located avoiding degenerated patterns.

Proposition 2. Matrix $\mathrm{M}(x)$ is regular if the particle distribution verifies


\section{Register for free at https//www.scipedia.com to download the version without the watermark}

Fig. 3. Substitution of a finite element node by one particle: non admissible distribution (left). Substitution of a finite element node by two particles: admissible distribution (right).

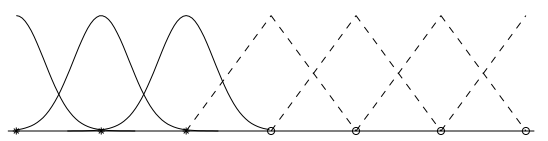

Fig. 4. Non admissible distribution. $\widetilde{\Omega}$ is under the influence one particle.

These restrictions are also valid for possible distributions of particles in a mixed interpolation. For instance, in a one-dimensional domain with an order one consistency (linear interpolation) a finite element node can not be replaced by a single particle, see figure 3 . Two particles, with dilation parameters large enough, are needed in order to ensure that everywhere in 
$\Omega^{\rho}$ the scalar product does not degenerate. Figure 3 depicts these situations. For each particle, its corresponding weighting function $\phi\left(\frac{x-x_{i}}{\rho}\right)$ is plotted.

Figure 4 also shows a non admissible distribution of particles. In the region where both particle and finite element interpolations have an influence, $\widetilde{\Omega}$, there are not enough particles (only one is present) to ensure the regularity of $\mathbf{M}(x)$. An obvious solution for this problem, maintaining the same particle distribution, is to chose a dilation parameter large enough, see figure 2.

\subsection{Convergence analysis}

It is easy to verify that the mixed interpolation proposed preserves the convergence rate of FEM and EFG. As an example, function $u(x)=x^{4}+2 x^{3}$ is interpolated for $x \in \bar{\Omega}=[-1,1]$. The three regions of influence of finite elements, particles and the mixed interpolation are: $\Omega^{h}=[-h, 1], \Omega^{\rho}=[-1,0]$ and $\widetilde{\Omega}=[-h, 0]$, where $h$ is the size of finite elements, see figure 2 .
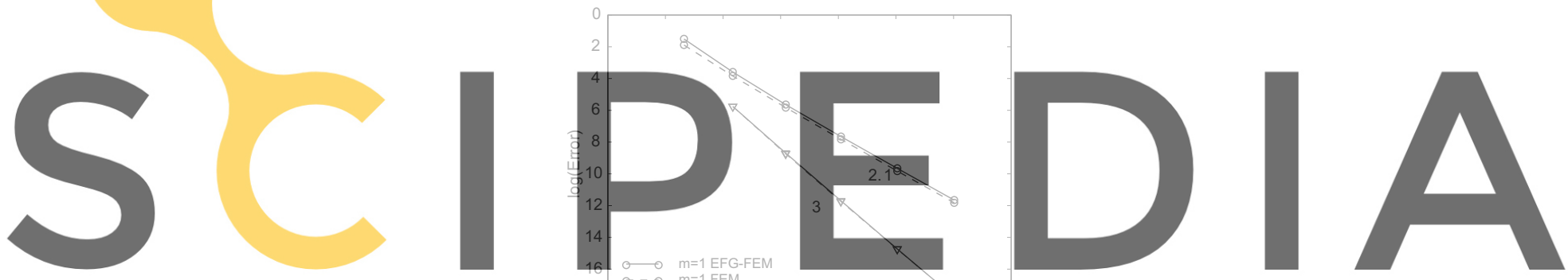



Fig. 5. Convergence of FEM and coupled FEM-EFG for a distribution of elements and particles shown in figures 2 and 6 .
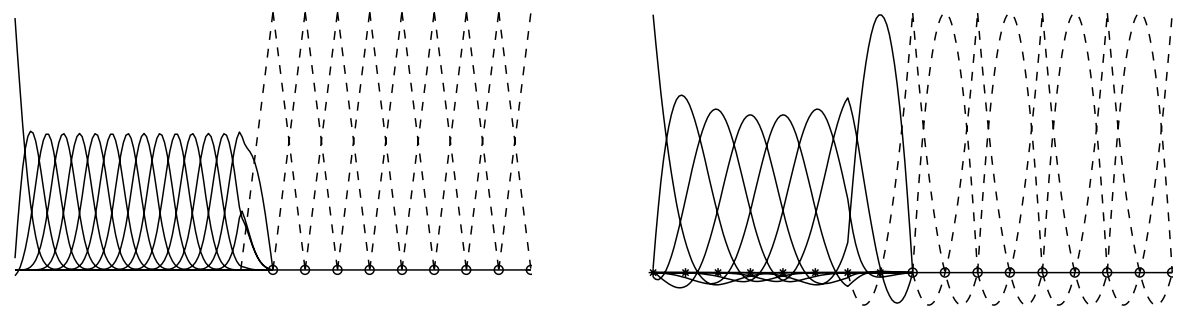

Fig. 6. Approximation functions with two particles in the transition region $\widetilde{\Omega}$ and consistency of order one (left) and order two (right).

Figure 5 shows the convergence rate - logarithm of the error in $L^{2}([-1,1])$ versus the total number of degrees of freedom - in two cases: standard linear 
finite elements and a coupled finite element-EFG approximation of order one. With this distribution of particles and with consistency of order one, this approach gives the same results as the one proposed by Belytschko et al. [4]. However, the mixed interpolation proposed here can be used with other distributions of elements and particles and any order of consistency, see figure 6 . See also in figure 5 the convergence results obtained with $m=2$ and the second particle distribution of figure 6 .

\section{Numerical example: convection-diffusion equation}

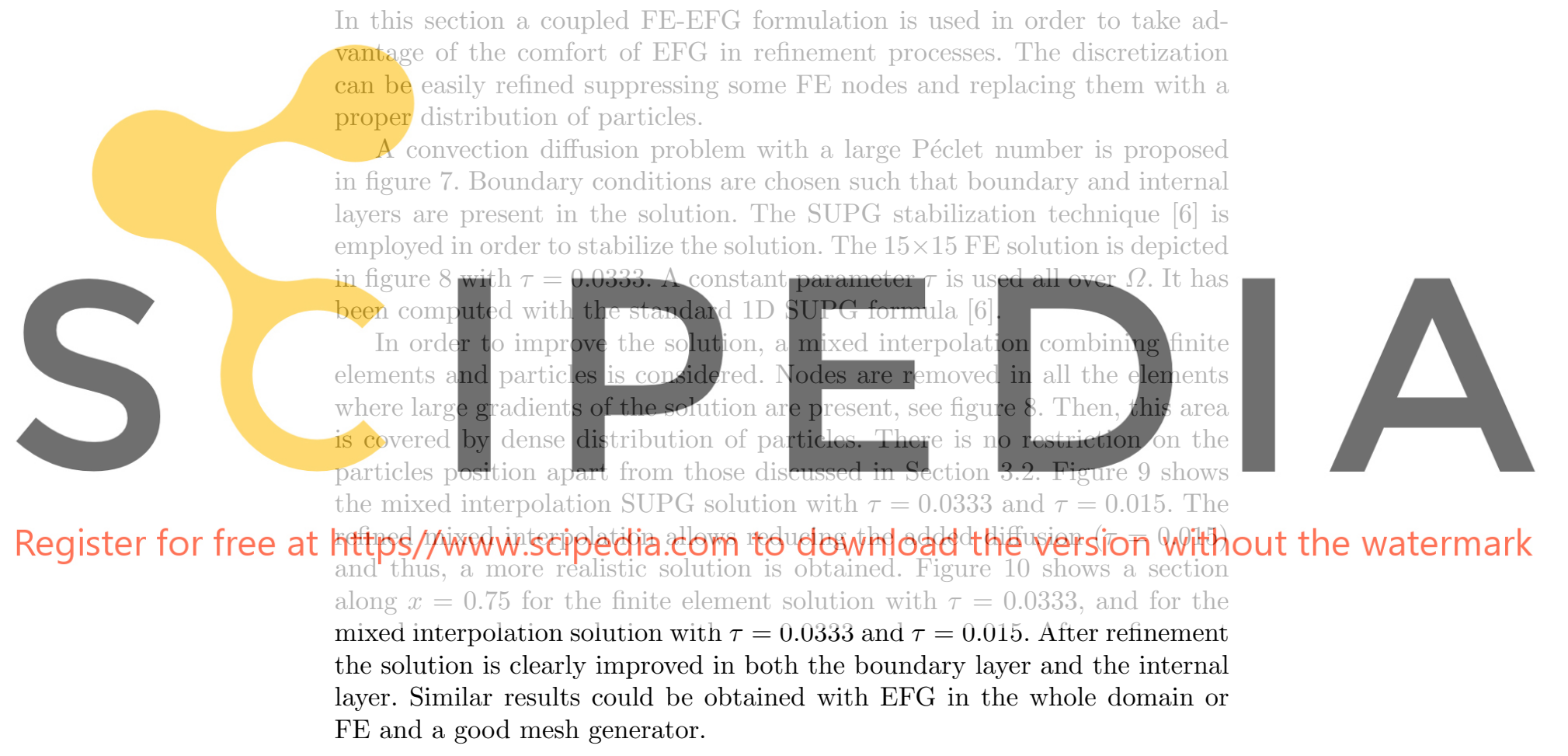

Remark 3. The consistent SUPG weak form for this problem can be written as: find $u$ such that

$$
\int_{\Omega}[\nabla v \nu \nabla u+(\mathbf{a} \cdot \nabla u)] \mathrm{d} \Omega+\sum_{e} \int_{\Omega_{e}} \tau(\mathbf{a} \cdot \nabla v)[-\nu \Delta u+\mathbf{a} \cdot \nabla u] \mathrm{d} \Omega=0
$$

where it is assumed that $v=0$ on $\partial \Omega$ in order to simplify the notation. In (16) one can observe the usual Galerkin terms, first term on l.h.s., and the extra SUPG stabilization terms, second term on l.h.s., integrated over the elements or integration cells, $\Omega_{e}$. Note that there is one extra term which includes second derivatives of the approximation, which can not be eliminated with 



integration by parts. When the EFG interpolation is used, second derivatives of the approximation are well defined in the whole domain and thus, the consistent weak form (16) can be considered. However, when linear finite elements are used, this term with second derivatives is usually neglected and this non-consistent weak form is actually used

$$
\int_{\Omega}[\nabla v \nu \nabla u+v(\mathbf{a} \cdot \nabla u)] \mathrm{d} \Omega+\sum_{e} \int_{\Omega_{e}} \tau(\mathbf{a} \cdot \nabla v)(\mathbf{a} \cdot \nabla u) \mathrm{d} \Omega=0
$$

The lack of consistency due to this neglected term leads to errors in the numerical scheme, see [10] for details. These errors are almost negligible for the stationary case: there are not substantial differences between the solution obtained with the mixed interpolation with the non-consistent weak form (17) and the solution obtained with the mixed interpolation with (17) in the FE region and (16) in the EFG region. However, this is not the case in transient 



Fig. 9. Coupled SUPG solution with $\tau=0.0333$ (left) and $\tau=0.015$ (right)



Fig. 10. Section along $x=0.75$ for the finite element interpolation with $\tau=0.0333$ and the coupled formualtion with $\tau=0.0333$ and $\tau=0.015$

problems, where the errors due to the lack of consistency can drastically degrade the accuracy and convergence in time, see [9].

\section{Concluding remarks}

This paper develops a mixed interpolation, it is based on finite element and mesh-free methods. Particles can be added arbitrarily in the region of the computational domain where the finite element interpolation is not complete. This ensures continuity of the solution (no coupling via Lagrange multipliers is imposed) and also enforces a uniform order of consistency (and thus of convergence) everywhere in the computational domain. The convergence properties of the mixed approximation are similar to those of the finite element method or EFG. For the sake of clarity, EFG has been used as the mesh-free method. However, generalization to other mesh-free methods is straight forward. 
The coupled FE-EFG formulation can be employed in order to take advantage of the comfort of EFG in refinement processes. The discretization can be easily refined just taking off some nodes and replacing them with a suitable distribution of particles. A convection-diffusion problem shows the applicability of the proposed formulation. Moreover, in this kind of problems, the continuity of the second derivatives of the EFG shape functions allows defining consistent stabilized formulations in the EFG region.

\section{References}

1. Belytschko T., Lu Y. Y. and Gu L. (1994) Element-free Galerkin methods. Int. J. Num. Meth. Engrg. 37, 229-256

2. Belytschko T., Krongauz Y., Fleming M., Organ D. and Liu W.K. (1996) Smoothing and accelerated computations in the element free galerkin method. J. Comp. Appl. Math. 74, 111-126

3. Belytschko T., Krongauz Y., Organ D., Fleming M. and Krysl P. (1996) Meshless Methods: an Overview and Recent Developments. Comp. Meth. Appl. Mech. Engrg. 139, 3-47

4. Belytschko T., Organ D. and Krongauz Y. (1995) A coupled finite element-free Galerkin method. Comput. Mech. 17, 186-195

5. Bonet J. and Kulasegaram S. (1999) Variational and momentum preservation aspects of smooth particle hydrodynamic formulations. Comp. Meth. Appl. Mech. Engrg. 180, 97-115

6. Brooks A.N. and Hugues T. (1982) Streamline upwind/petrov-galerkin formulations for convection dominated flows with particular emphasis on the incompressible navier-stokes equations. Comput. Meth. Appl. Mech. Engrg. 32, 259

7. Hegen D. (1996) Element Free Galerkin methods in combination with finite element approaches. Comp. Meth. Appl. Mech. Engrg. 135, 143-166

8. Huerta A. and Fernández-Méndez S. (2001) Enrichment and coupling of the finite element method and meshless methods. Int. J. Num. Meth. Engrg., 48, $1615-1636$

9. Huerta A. and Fernández-Méndez S. (2001) Time accurate consistently stabilized mesh-free methods for convection dominated problems. Int. J. Num. Meth. Engrg., submitted

10. Jansen K.E., Collins S.S., Whiting C. and Shakib F. (1999), A better consistency for low-order stabilized finite element methods. Comput. Meth. Appl. Mech. Engrg. 174, 153-170

11. Liu W.K., Belytschko T. and Oden J.T. eds. (1996) Meshless Methods. Comput. Meth. Appl. Mech. Engrg. 139

12. Liu W.K., Li S. and Belytschko T. (1997) Moving least square reproducing kernel methods. (I) Methodology and convergence. Comput. Methods Appl. Mech. Engrg. 143, 113-154 